Bull. Korean Math. Soc. 50 (2013), No. 3, pp. 801-809

http://dx.doi.org/10.4134/BKMS.2013.50.3.801

\title{
A CONSTRUCTION OF COMMUTATIVE NILPOTENT SEMIGROUPS
}

\author{
Qiong Liu, Tongsuo Wu, And Meng Ye
}

\begin{abstract}
In this paper, we construct nilpotent semigroups $S$ such that $S^{n}=\{0\}, S^{n-1} \neq\{0\}$ and $\Gamma(S)$ is a refinement of the star graph $K_{1, n-3}$ with center $c$ together with finitely many or infinitely many end vertices adjacent to $c$, for each finite positive integer $n \geq 5$. We also give counting formulae to calculate the number of the mutually non-isomorphic nilpotent semigroups when $n=5,6$ and in finite cases.
\end{abstract}

\section{Introduction}

The present paper is motivated by works on the zero-divisor graph, which was first defined and studied for commutative rings by I. Beck in [2] and later modified and further studied by D. F. Anderson and P. S. Livingston in [1]. In 2002, DeMeyer, Mckenzie and Schneider began the study of zero-divisor graph of a commutative semigroup with 0 in [6]. Since then, much work has been done and this becomes a lively branch in semigroup theory and graph theory, see e.g. $[4,5,10,11,12]$.

For a commutative semigroup $S$ with zero element 0 (i.e., $0 S=0$ ), the zero-divisor graph of $S$ denoted by $\Gamma(S)$, is a simple undirected graph. The vertex set is $Z(S)^{*}$, the set of all nonzero zero-divisors of $S$, where for distinct $x, y \in Z(S)^{*}$, there is an edge connecting $x$ and $y$ if and only if $x y=0$.

For notions and results about commutative semigroups, we use [7] as a basic reference. We adopt the graph theoretic notations from [3]. Especially we use $K_{1, n}$ to denote the star graph (i.e., a special complete bipartite graph). Recall that a simple graph $G$ is called a refinement of a connected simple graph $H$ if $V(G)=V(H)$ and $a-b$ in $H$ implies $a-b$ in $G$ for all distinct vertices of $G$, where $a-b$ means that $a \neq b$ and $a$ is adjacent to $b$. A vertex $c$ is called

Received February 25, 2012; Revised July 2, 2012

2010 Mathematics Subject Classification. Primary 20M14; Secondary 05C90.

Key words and phrases. nilpotent semigroup, refinement of a star graph, structure, counting formula.

This research is partly supported by the Natural Science Foundation of Shanghai (No.10ZR1412500) and Youth Foundation of Shanghai (No.sdl10017).

This research is partly supported by the National Natural Science Foundation of China (No.11271250). 
a center of a graph $G$ if $c$ is adjacent to every vertex of $G$. In [4, Theorem $3(3)$ ], it was proved that any refinement of a star graph has a corresponding semigroup.

Throughout this paper, for any vertex $c$ in a graph $G$, let $G_{c}^{*}$ be the subgraph of $G$ induced on the following vertex set

$$
V(G) \backslash\{u \in V(G) \mid u=c \text { or } u \text { is an end vertex adjacent to } c\} \text {. }
$$

In this paper, we construct nilpotent semigroup $S$ such that $S^{n}=\{0\}, S^{n-1} \neq$ $\{0\}$ and the zero-divisor graph $\Gamma(S)$ is a refinement of a star graph $K_{1, n-3}$ together with arbitrarily many end vertices adjacent to the center, where $n \geq 5$. We also prove that there is (up to isomorphism) exactly one nilpotent semigroup satisfying this condition. With the aid of the detailed structure results just mentioned, we are able to give a counting formula for calculating the number of mutually non-isomorphic nilpotent semigroups $S$ such that $\Gamma(S) \cong K_{1, n-3}$, when $n=5,6$ and $\Gamma(S)$ is a finite graph.

Now we record a general result on the centers of a refinement of a star graph. Recall that an ideal $I$ of $S$ is called an annihilating ideal if $I=A n n(x)$ for some $x \in S$.

Proposition 1.1. For any semigroup $S$ with 0 whose zero-divisor graph $\Gamma(S)$ is a refinement of a star graph with $c$ as one of its centers, either $c^{2}=c$ or $c^{2}=0$ holds.

Proof. Let $Z(S)$ be the set of all zero-divisors of $S$. Assume further that $Z(S)$ is not an annihilating ideal of $S$. Then $Z(S) \backslash\{c\}=\operatorname{Ann}(c) \unlhd S$ and $c^{2} \neq 0$. Clearly, we can see that $A n n(c)$ is a maximal annihilating ideal of $S$, thus $A n n(c)$ is a prime ideal of $S$. We assert $c^{2}=c$. In fact if $c^{2} \neq c$, then $c^{2} \in \operatorname{Ann}(c)$. Since $\operatorname{Ann}(c)$ is a prime ideal of $S$, we obtain $c \in \operatorname{Ann}(c)$. Thus $c^{2}=0$, a contradiction. If $Z(S)$ is an annihilating ideal of $S$, then $Z(S)=\operatorname{Ann}(c)$ and therefore $c^{2}=0$.

Corollary 1.2. Assume that $S$ is a nilpotent semigroup whose zero-divisor graph $\Gamma(S)$ is a refinement of a star graph with a center $c$. Then $c^{2}=0$.

\section{Main result}

Theorem 2.1. For any finite integer $n \geq 5$, let $m=n-3$. For any finite or infinite set $T$ with $|T| \geq 1$, we fix an element $t_{0} \in T$. Let $S=\{0, c\} \cup$ $\left\{a_{1}, a_{2}, \ldots, a_{m}\right\} \cup T$ be a disjoint union of three subsets. Define in $S$ a binary operation by $0 S=S 0=\{0\}=c S=S c$ and the following:

$$
T^{2}=\left\{a_{m}\right\}, a_{i} T=\left\{a_{i-1}\right\}=T a_{i}, a_{i} a_{j}=a_{i-1} a_{j+1}, \forall 1 \leq i, j \leq m,
$$

where we order $a_{m+1}=t_{0}$ and $a_{0}=c$, so that $a_{i} a_{m}=a_{i-1} t_{0}, a_{1} t=c, a_{1} a_{j}=$ $c a_{j+1}=0$. Then $S$ is a commutative semigroup with zero element 0 such that

(1) $a_{i} \in S^{n-i-1} \backslash S^{n-i}(\forall 1 \leq i \leq m)$. In particular, $S^{n-1} \neq\{0\}$ and $S^{n}=\{0\}$. 
(2) $\Gamma(S)=\left(G_{n} \cup T\right)+\{c\}$ and $\Gamma(S)_{c}^{*}=G_{n}$, where $\left|V\left(G_{n}\right)\right|=n-3$ and $G_{n}$ is the subgraph induced on $\left\{a_{1}, a_{2}, \ldots, a_{m}\right\}$. In particular, $\Gamma(S)$ is a refinement of $K_{1, n-3}$ with center $c$ together with finitely many or infinitely many end vertices adjacent to the center $c$.

Proof. First, the multiplication defined in $S$ is clearly well defined. Without loss of generality, assume $1 \leq i \leq j \leq m$. Then $a_{j} a_{i}=a_{j-(j-i)} a_{i+(j-i)}=a_{i} a_{j}$ and it follows from the construction that the law of commutativity holds in $S$.

Second, we need to verify the associativity of the binary operation defined on $S$. Without loss of generality, it is only necessary to test the case $|T|=2, m=$ 3. Then $S=\left\{0, c, a_{1}, a_{2}, a_{3}, t_{1}, t_{2}\right\}$, and we have the following multiplication table: The set $\left\{a_{2}, t_{1}, t_{2}\right\}$ is a generating set of $S$ under the multiplication table

\section{TABLE 1}

\begin{tabular}{c|ccccccc}
$\cdot$ & 0 & $c$ & $a_{1}$ & $a_{2}$ & $a_{3}$ & $t_{1}$ & $t_{2}$ \\
\hline 0 & 0 & 0 & 0 & 0 & 0 & 0 & 0 \\
$c$ & 0 & 0 & 0 & 0 & 0 & 0 & 0 \\
$a_{1}$ & 0 & 0 & 0 & 0 & 0 & $c$ & $c$ \\
$a_{2}$ & 0 & 0 & 0 & 0 & $c$ & $a_{1}$ & $a_{1}$ \\
$a_{3}$ & 0 & 0 & 0 & $c$ & $a_{1}$ & $a_{2}$ & $a_{2}$ \\
$t_{1}$ & 0 & 0 & $c$ & $a_{1}$ & $a_{2}$ & $a_{3}$ & $a_{3}$ \\
$t_{2}$ & 0 & 0 & $c$ & $a_{1}$ & $a_{2}$ & $a_{3}$ & $a_{3}$
\end{tabular}

above, since $0=a_{2}^{2}, c=a_{2} t_{1}^{2}, a_{1}=a_{2} t_{1}, a_{3}=t_{1}^{2}$. By Light's associativity test (see [8]), it is enough to verify that $x(u y)=(x u) y$ for all $x, y \in S \backslash\{0, c\}$, and $u$ is any element in the generating set of $S$. Observe the following two multiplication tables: where the header row of Table 2 is the values of $a_{2} a_{1}, a_{2}^{2}, a_{2} a_{3}, a_{2} t_{1}, a_{2} t_{2}$,

\section{TABLE 2}

\begin{tabular}{c|ccccc}
$\cdot$ & 0 & 0 & $c$ & $a_{1}$ & $a_{1}$ \\
\hline$a_{1}$ & 0 & 0 & 0 & 0 & 0 \\
$a_{2}$ & 0 & 0 & 0 & 0 & 0 \\
$a_{3}$ & 0 & 0 & 0 & 0 & 0 \\
$t_{1}$ & 0 & 0 & 0 & $c$ & $c$ \\
$t_{2}$ & 0 & 0 & 0 & $c$ & $c$
\end{tabular}

where the header column of Table 3 is the values of $a_{1} a_{2}, a_{2}^{2}, a_{3} a_{2}, t_{1} a_{2}, t_{2} a_{2}$. We can see that the entries in the various cells in Table 2 agrees with the entries in the corresponding cells of Table 3 . This shows that $x\left(a_{2} y\right)=\left(x a_{2}\right) y$ for all $x, y \in S \backslash\{0, c\}$. Thus $x\left(a_{2} y\right)=\left(x a_{2}\right) y$ holds for all $x, y$ in $S$. Similarly, we have $x\left(t_{1} y\right)=\left(x t_{1}\right) y$ and $x\left(t_{2} y\right)=\left(x t_{2}\right) y$ for all $x, y$ of $S$. By Light's test, the law of associativity holds for $S$. Thus $S$ is a commutative semigroup with zero element 0 . 
TABLE 3

\begin{tabular}{c|ccccc}
$\cdot$ & $a_{1}$ & $a_{2}$ & $a_{3}$ & $t_{1}$ & $t_{2}$ \\
\hline 0 & 0 & 0 & 0 & 0 & 0 \\
0 & 0 & 0 & 0 & 0 & 0 \\
$c$ & 0 & 0 & 0 & 0 & 0 \\
$a_{1}$ & 0 & 0 & 0 & $c$ & $c$ \\
$a_{1}$ & 0 & 0 & 0 & $c$ & $c$
\end{tabular}

Third, since $T^{2}=\left\{a_{m}\right\}, a_{1} T=\{c\}$ and $a_{i} T=\left\{a_{i-1}\right\}$ for all $2 \leq i \leq m$, we obtain by induction $T^{p}=\left\{a_{m-p+2}\right\}=\left\{a_{n-p-1}\right\}$ for all $2 \leq p<n-1$. In particular for $p=n-1$ we have $T^{n-1}=\{c\}$. Hence $S^{p} \neq\{0\}$ since $T^{p} \subseteq S^{p}$ for all $p<n$, and $S^{n-1}=\{0, c\}$. Thus $S^{n}=\{0\}, S^{n-1}=\{0, c\}$. Obviously, each vertex in $T$ is an end vertex adjacent to the center vertex $c$, and $\Gamma(S) \backslash T$ is a refinement of the star graph $K_{1, m}$.

Finally, we claim that $a_{i} \in S^{n-i-1} \backslash S^{n-i}$ holds for each $i$ with $1 \leq i \leq m$. In fact, if this is not the case, then assume that $j$ is the least number such that $a_{j} \in S^{n-j}$. Then for all $i<j$ we have $a_{i} \in S^{n-i-1} \backslash S^{n-i}$. Clearly, $j \geq 2$ and hence such $i$ exists. By calculation, we have $S^{n-i-1}=S^{n-i} \cup\left\{a_{i}\right\}$ for all $1 \leq i \leq m$. Let $i=j-1$, then $S^{n-j}=S^{n-j+1} \cup\left\{a_{j-1}\right\}$. Thus $a_{j} \in S^{n-j+1}$ since $a_{j} \in S^{n-j}$ and $a_{j} \neq a_{j-1}$. Let $i=j-2$, then $S^{n-j+1}=S^{n-j+2} \cup\left\{a_{j-2}\right\}$. Thus $a_{j} \in S^{n-j+2}$ since $a_{j} \in S^{n-j+1}$ and $a_{j} \neq a_{j-2}$. Continuing this process until we get $a_{j} \in S^{n-1}$. Note that $S^{n-1}=\{0, c\}$, a contradiction. The contradiction shows that $a_{i} \in S^{n-i-1} \backslash S^{n-i}$ holds for all $1 \leq i \leq m$.

From the proof above, it is easy to prove that the graph $G$ constructed in Theorem 2.1 has a unique nilpotent semigroup $S$ such that $S^{n}=\{0\}, S^{n-1} \neq$ $\{0\}$ and $\Gamma(S)=\left(G_{n} \cup T\right)+\{c\}$.

Remark 2.2. For $n=5$ and $n=6$, we draw the corresponding graphs in the following Figure 1 and Figure 2, respectively. It can be verified that for the graph $G$ in Figure 1 (respectively, in Figure 2), if $S^{k}=\{0\}$ for some $k$ and $\Gamma(S)=G$, then $S^{5}=\{0\}\left(S^{6}=\{0\}\right.$ respectively). Also there exists a unique nilpotent semigroup $S$ such that $S^{5}=\{0\}, S^{4} \neq\{0\}$ (respectively, $\left.S^{6}=\{0\}, S^{5} \neq\{0\}\right)$ and $\Gamma(S)=G$.

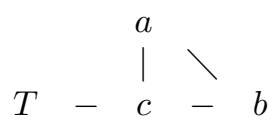

FIGURE 1 


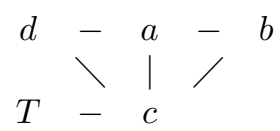

FIGURE 2

\section{Counting semigroups}

In the following, we will consider the number of non-isomorphic corresponding semigroups of the graph $G$ in Figure 1 and Figure 2. First, we give some known structural results which we have obtained in [9].

Lemma 3.1. For a semigroup $S$, the following statements are equivalent:

(1) $S$ is a semigroup such that $S^{4}=\{0\}, S^{3} \neq\{0\}$ and $\Gamma(S)=\left(K_{1,1} \cup T\right)+$ $\{c\}$, where $|T| \geq 1$ and $\Gamma(S)_{c}^{*}=K_{1,1}$.

(2) $S=\{0, c, a, b\} \cup T$ is a disjoint union of two subsets, $c S=\{0\}=0 S, a b=$ $0=a^{2},\{a, b\} T=\{c\}$ and exactly one of the following conditions holds:

(i) $b^{2}=0$ and $T^{2}=\{a, b\}$, where $|T| \geq 2$. In this case, $S$ is a disjoint union of $S^{2}$ and $T$, where $S^{2}=\{0, c, a, b\}$.

(ii) $b^{2} \in\{0, c\}$ and $T^{2}=\{a\}$, where $|T| \geq 1$. In this case, $S$ is a disjoint union of $S^{2},\{b\}$ and $T$, where $S^{2}=\{0, c, a\}$.

Lemma 3.2. For any semigroup $S$, the following statements are equivalent:

(1) $S$ is a semigroup such that $S^{5}=\{0\}, S^{4} \neq\{0\}$ and $\Gamma(S)=\left(K_{1,2} \cup T\right)+$ $\{c\}$, where $|T| \geq 1$ and $\Gamma(S)_{c}^{*}=K_{1,2}$.

(2) $S=\{0, c, a, b, d\} \cup T$ is a disjoint union of two subsets, $c S=\{0\}=0 S$, $a b=a d=0, b d=b^{2}=c, a^{2}=0, a T=\{c\},\{b, d\} T=\{a\}$ and exactly one of the following conditions holds:

(i) $d^{2}=c$, and $T^{2}=\{b, d\}$. This is the case only when $|T| \geq 2$. In this case, $S$ is a disjoint union of $S^{2}$ and $T$, where $S^{2}=\{0, c, a, b, d\}$ and $S^{3}=\{0, c, a\}$.

(ii) $d^{2} \in\{0, c\}$ and $T^{2}=\{b\}$. In this case, $S$ is a disjoint union of $S^{2},\{d\}$ and $T$, where $S^{2}=\{0, c, a, b\}$ and $S^{3}=\{0, c, a\}$.

Lemma 3.3. For any semigroup $S$, the following statements are equivalent:

(1) $S$ is a semigroup such that $S^{4}=\{0\}, S^{3} \neq\{0\}$ and $\Gamma(S)=\left(K_{1,2} \cup T\right)+$ $\{c\}$, where $|T| \geq 1$ and $\Gamma(S)_{c}^{*}=K_{1,2}$.

(2) $S=\{0, c, a, b, d\} \cup T$ is a disjoint union of two subsets, where $|T| \geq 1$, $c S=\{0\}=0 S, a b=a d=0, a^{2}=0, b d=c,\{a, b\} T=\{c\}$ and exactly one of the following conditions holds:

(i) $b^{2}=0$, and either $d^{2} \in\{0, c\}, d T=\{a\}, T^{2}=\{b\} ;$ or $d^{2}=a, d T=$ $\{b\}, T^{2}=\{b\}$. In each subcase, $S^{2}=\{0, c, a, b\}$.

(ii) $\left\{b^{2}, d^{2}\right\} \subseteq\{0, c\}, d T=\{c\}$ and $T^{2}=\{a\}$. In this case, $S^{2}=\{0, c, a\}$.

Let $X=\{i \mid 1 \leq i \leq n\}$ and $Y=\{0,1\}$. Let $H$ be the cyclic group of order two which is generated by the permutation $(0,1)$. Let $F$ be the set of all maps 
from $X \times X$ to $Y$. Define in $F$ two equivalent relations respectively in the following ways:

(i) $h \sim_{2} k$ if and only if there exists a permutation $\sigma \in S_{n}$ such that $h(i, j)=k(\sigma(i), \sigma(j))$ for all $1 \leq i, j \leq n$.

(ii) $h \sim_{1} k$ if and only if there exists a permutation $\sigma \in S_{n}$ and a permutation $\tau \in H$ such that $h(i, j)=\tau k(\sigma(i), \sigma(j))$ for all $1 \leq i, j \leq n$.

Let $A(n)$ and $B(n)$ be the number of equivalent classes relative to $\sim_{1}$ and $\sim_{2}$, respectively. Recall that in $S_{n}$ two permutations are conjugate if and only if they have the same type when decomposed into products of mutually disjoint cyclic permutations. Thus Burnside's Lemma could be used to calculate the $B(n)$. We have a program to compute the $B(n)$ via a computer for all $n \leq 50$. When $4 \nmid n$, we observe that $A(n)=\frac{B(n)}{2}$.

Another way to interpret the numbers $A(n)$ and $B(n)$ is illustrated in the following. Consider the following graphs with the given set $X$ of $n$ vertices. Each vertex is allowed to have at most one loop and every pair of distinct vertices can have at most one edge. Then the largest such graph is the complete graph $K_{n}$ together with $n$ loops. We denote this graph as $K_{n}^{\circ}$. Then each $f$ in $F$ is just the adjacency matrix of a corresponding graph $G_{f}$. Thus $B(n)$ is just the number of non-isomorphic such graphs obtained on the given set of $n$ vertices. For $\tau=(0,1)$, notice that $\tau(f)$ corresponds to the complement graph of $G_{f}$ in $K_{n}^{\circ}$. In the following Figure $3, G=K_{3}^{\circ}$ and $H$ is the complement graph of $L$ in $K_{3}^{\circ}$.

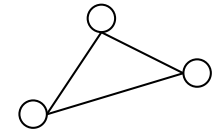

G

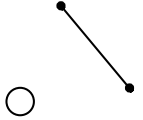

$\mathrm{H}$

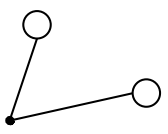

$\mathrm{L}$

FIGURE 3

This gives another explanation to the number $A(n)$. Let $E$ be the set of all subgraphs $G$ of $K_{n}^{\circ}$ such that $G$ has the $n$ vertices. Define in $E$ an equivalent relation in the following way: $G_{1}$ and $G_{2}$ are equivalent if and only if either $G_{1} \cong G_{2}$ or $G_{1}$ is isomorphic to the complement of $G_{2}$ in $K_{n}^{\circ}$. Then $A(n)$ is the number of the equivalent classes.

Theorem 3.4. (1) For any finite set $T$ with $|T| \geq 2$, let $G=\left(K_{1,1} \cup T\right)+\{c\}$ be a graph with $G_{c}^{*}=K_{1,1}$. Then $G$ has $f(n)$ mutually non-isomorphic nilpotent semigroups, where $f(n)=A(n)+3 n+5, n=|T|$.

(2) The graph $K_{3}+1$ (i.e., $\left.\left(K_{1,1} \cup\{b\}\right)+\{c\}\right)$ has 9 mutually non-isomorphic nilpotent semigroups.

Proof. (1) Assume $|T| \geq 2$ and let $S$ be a nilpotent semigroup whose zerodivisor graph is $\Gamma(S)=\left(K_{1,1} \cup T\right)+\{c\}$. By Theorem 2.1, $S^{5}=\{0\}$ and there 
is exactly one semigroup $S$ such that $S^{5}=\{0\}$ and $S^{4} \neq\{0\}$. From Lemma 3.1 we can see that in case (i), $S$ is completely determined by the $t t^{\prime}$ for all $t, t^{\prime} \in T$, which could be freely chosen from the set $\{a, b\}$ such that $T^{2}=\{a, b\}$. In case (ii), $S$ is completely determined by $b^{2}$, which is 0 or $c$. Hence, when $S^{4}=\{0\}$ and $S^{3} \neq\{0\}$, the number of mutually non-isomorphic nilpotent semigroups is $(A(n)-1)+2$, where $n \geq 2$. The remaining case is $S^{3}=\{0\}, S^{2}=\{0, c\}$. By hypothesis, there exist distinct elements $a, b \in S \backslash\{0, c\}$ such that $a b=0$. Clearly, $a, b$ are symmetric. In this case, $S$ is completely determined by the vector $\left(a^{2}, b^{2}\right)$ which could be one of the $(0,0),(c, 0),(c, c)$, and the number of end vertices adjacent to $c$ whose square is $c$. Thus the number of mutually non-isomorphic nilpotent semigroups is $3(n+1)$. Therefore, the total number of mutually non-isomorphic nilpotent semigroups is $f(n)=A(n)+3 n+5$, when $n \geq 2$.

(2) In this case, we can see that $|T|=1$. Thus case (i) of Lemma 3.1 does not exist. Similar to (1) observation, we can easily find that the total number of mutually non-isomorphic nilpotent semigroups is 9 when $\Gamma(S)=K_{3}+1$.

Theorem 3.5. (1) Suppose $G=\left(K_{1,2} \cup T\right)+\{c\}$ is a graph satisfying $G_{c}^{*}=$ $K_{1,2}$, where $|T| \geq 2$. Then $G$ has $g(n)$ mutually non-isomorphic nilpotent semigroups, where $g(n)=A(n)+6 n+14$ and $n=|T|$.

(2) The graph $\left(K_{1,2} \cup\{b\}\right)+\{c\}$ has 21 mutually non-isomorphic nilpotent semigroups.

Proof. (1) Assume $|T| \geq 2$ and let $S$ be a nilpotent semigroup such that $\Gamma(S)=$ $\left(K_{1,2} \cup T\right)+\{c\}$. By Theorem 2.1, there is exactly one nilpotent semigroup $S$ with $S^{6}=\{0\}$ and $S^{5} \neq\{0\}$. Then, there are still three remaining cases: (1) $S^{5}=\{0\}$ but $S^{4} \neq\{0\} ;$ (2) $S^{4}=\{0\}$ but $S^{3} \neq\{0\}$; (3) $S^{3}=\{0\}$ but $S^{2} \neq\{0\}$. When $S^{5}=\{0\}, S^{4}=\{0, c\}$, by Lemma 3.2, we find that in case (i), $S$ is completely determined by an $n \times n$ matrix taken from in $T^{2}$, where $T^{2}=\{b, d\}$. In case (ii), we easily know that the number is 2 . So in this case, the number of mutually non-isomorphic nilpotent semigroups is $(A(n)-1)+2$, where $n \geq 2$. When $S^{4}=\{0\}, S^{3}=\{0, c\}$, from the multiplication formula we have given in Lemma 3.3 we can easily get that in case (i), the number is 3 . While in case (ii), the number is also 3 . Hence, in this condition, the total number of mutually non-isomorphic nilpotent semigroups is 6. Finally, we will discuss $S^{3}=\{0\}, S^{2}=\{0, c\}$. By assumption, there exist distinct elements $a, b, d \in S \backslash\{0, c\}$ such that $a b=a d=0, b d \neq 0$. Obviously, $b, d$ are symmetric. $S$ is completely determined by $a^{2}, b^{2}, d^{2}$ and the number of end vertices in $T$ whose square is $c$. So in this case, the number of mutually non-isomorphic nilpotent semigroups is $2 \times 3 \times(n+1)=6(n+1)$. To conclude, the total number of mutually non-isomorphic nilpotent semigroups is $g(n)=1+(A(n)-1)+2+6+6(n+1)=A(n)+6 n+14$, when $n \geq 2$.

(2) When $|T|=1$, we can see that Lemma 3.2(i) does not exist. So the total number of mutually non-isomorphic nilpotent semigroups is 21 , follows directly from the argument of (1). 
In the end of the paper, we list the first six values for $B(n), A(n), f(n)$ and $g(n)$ respectively in the following table:

TABLE 4

\begin{tabular}{|c|c|c|c|c|c|c|}
\hline$n$ & 1 & 2 & 3 & 4 & 5 & 6 \\
\hline$B(n)$ & 0 & 6 & 20 & 90 & 544 & 5096 \\
\hline$A(n)$ & 0 & 3 & 10 & 46 & 272 & 2548 \\
\hline$f(n)=A(n)+3 n+5(n \geq 2)$ & 9 & 14 & 24 & 63 & 292 & 2571 \\
\hline$g(n)=A(n)+6 n+14(n \geq 2)$ & 21 & 29 & 42 & 84 & 316 & 2598 \\
\hline
\end{tabular}

\section{References}

[1] D. F. Anderson and P. S. Livingston, The zero-divisor graph of a commutative ring, J. Algebra 217 (1999), no. 2, 434-447.

[2] I. Beck, Coloring of commutative rings, J. Algebra 116 (1988), no. 1, 208-226.

[3] F. Buckley and M. Lewinter, A Friendly Introduction to Graph Theory, Prentice-Hall, 2003.

[4] F. R. DeMeyer and L. DeMeyer, Zero-divisor graphs of semigroups, J. Algebra 283 (2005), no. 1, 190-198.

[5] L. DeMeyer, Y. J. Jiang, C. Loszewski, and E. Purdy, Classification of commutative zero-divisor semigroup graphs, Rocky Mountain J. Math. 40 (2010), no. 5, 1481-1503.

[6] F. R. DeMeyer, T. McKenzie, and K. Schneider, The zero-divisor graphs of a commutative semigroup, Semigroup Forum 65 (2002), no. 2, 206-214.

[7] P. A. Grillet, Commutative Semigroups, Kluwer Academic Publishers, DordrechtBoston-London, 2001.

[8] N. Kehayopulu and A. Philip, An algorithm for Light's associativity test using Mathematica, J. Comput. Inform. 3 (1993), no. 1, 87-98.

[9] Q. Liu, Nilpotent semigroups whose zero-divisor graph is a refinement of a star graph, J. Shanghai University of Electric Power 26 (2010), 305-307.

[10] T. S. Wu and L. Chen, Simple graphs and commutative zero-divisor semigroups, Algebra Colloq. 16 (2009), no. 2, 211-218.

[11] T. S. Wu and F. Cheng, The structures of zero-divisor semigroups with graph $K_{n} \circ K_{2}$, Semigroup Forum 76 (2008), no. 2, 330-340.

[12] T. S. Wu and D. C. Lu, Sub-semigroups determined by the zero-divisor graph, Discrete Math. 308 (2008), no. 22, 5122-5135.

QIONG LIU

Department of Mathematics

Shanghai University of Electric Power

Shanghai 200090, P. R. China

E-mail address: sky200547@126.com

Tongsuo Wu

Department of Mathematics

Shanghai Jiaotong University

Shanghai 200240, P. R. China

E-mail address: tswu@sjtu.edu.cn 
MENG YE

Department of Mathematics

Shanghai JiaOtong University

Shanghai 200240, P. R. China

E-mail address: sjtuyemeng@163.com 